\title{
On-line Rock Fragment Delineation on Simple Thresholding with Fuzzy Comprehensive Evaluation
}

\author{
Wang Weixing, Liang Yanjie, Liao Yipeng and Bai Lina \\ College of Physics and Information Engineering, Fuzhou University, \\ Fuzhou \\ 35002, China
}

\begin{abstract}
The classification and measurement of rock fragments is very important in mining and construction engineering. The monitoring system acquires and analyses the fragment images from a gravitational falling stream at the end of a moving conveyor belt, and the key function of the system is to construct an image segmentation algorithm. To achieve this goal, an adaptive thresholding algorithm with fuzzy comprehensive evaluation is proposed. Firstly, a grabbed image is roughly segmented by using a global auto-thresholding algorithm. Then each of the objects is measured and analyzed if it includes the multiple fragments touching each other, based on the fuzzy comprehensive evaluation method in which the salient fragment features of area, perimeter, shape, gradient magnitude and gray-level flatness are extracted, and for each of the features, the membership function is constructed experimentally. Finally, each of the touching fragment regions, as one image, is auto-thresholded again, and this procedure is repeated until no region can be further separated. The experimental results show that compared to cluster analysis, graph based, and FCM image segmentation algorithms the new algorithm can make image segmentation well for the falling fragments on-line.
\end{abstract}

Keyword: Rock fragment image, thresholding, fuzzy comprehensive evaluation

\section{Introduction}

The distributions of rock fragment size and shape are important indicators to evaluate the production quality. The traditional measurement by manual and sieving is time-consuming, limited sampling, inefficient and laborious. To overcome these disadvantages, many image processing methods have been used for the measurement and analysis of rock fragments on-line since 1984 [1-4]. As a general on-line monitoring system, the image acquisition sub-system is setup with a CCD camera either over a moving conveyor belt or at the end of a conveyor belt. In the former situation, the overlapping of the fragments makes image segmentation difficult, in the later situation; the fragments in a gravitational falling stream are easily delineated. For the falling stream situation, the sky can be viewed as the image background and most of the fragments can be separated during the falling process. Therefore, a simple thresholding algorithm is possibly used for the fragment detection in real time, but there is a consequent problem to split the touching fragment clusters.

Anyhow, to meet the on-line demand of the rock fragment measurement and analysis, a thresholding algorithm is the better choice for image segmentation. Generally, a global thresholding algorithm can obtain good results on images in which the gray level difference between objects and background is obvious. However, for the falling rock fragment images, due to the factors such as weather variation, dust, fragment touching, shadows and other noise, it is hard to segment image completely. To solve this problem, the paper proposes an adaptive threshold algorithm based on fuzzy comprehensive evaluation. In this algorithm, firstly, it segments a gray level image by using a global 
thresholding algorithm which is evaluated and selected based on five widely used thresholding algorithms. Then, it adopts the thresholding algorithm on the touching fragment regions separately, the result will either meet the criterion or acquire abnormal size, shape, gray level difference and gradient magnitude. Finally, for the latter situation, it will repeat the operations until no fragment touching object can be separated further. The algorithm flow chart is shown in Figure 1.

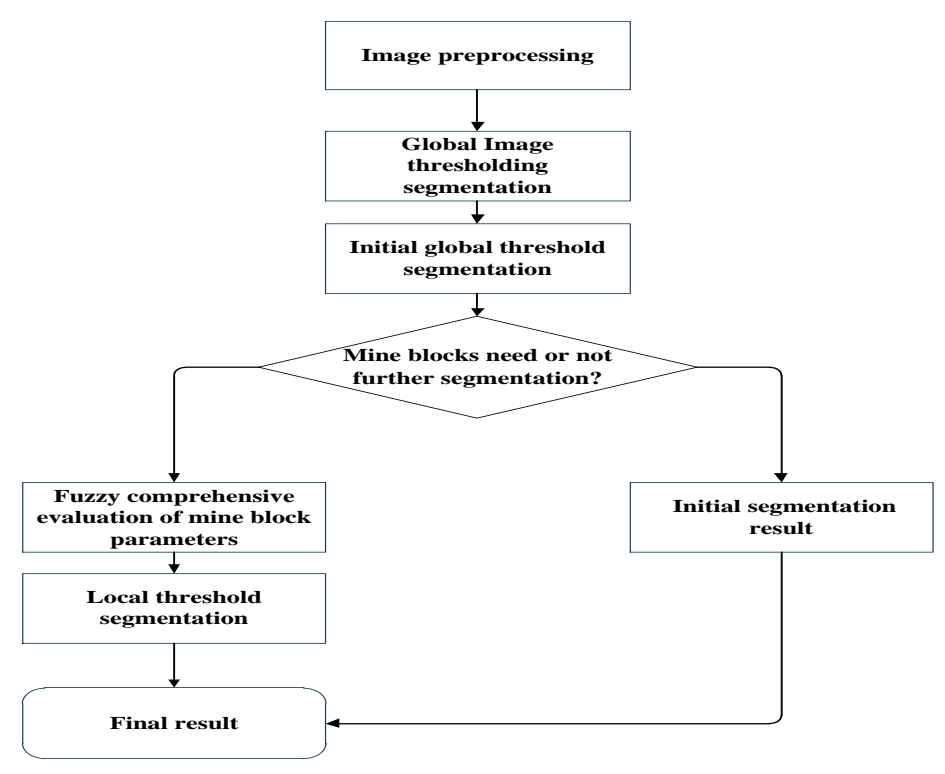

Figure 1. Procedure of Adaptive Thresholding Algorithm on Fuzzy Comprehensive Evaluation For A Rock Fragment Image

\section{Image Preprocessing and Initial Segmentation}

\subsection{Image Preprocessing}

Rock fragment images collected from a conveyor belt are RGB true color images. To reduce the processing data, the color images are converted into gray-scale images. Since the sky is as the background, the blue color dominates the images. According to RGB values of true color images and experience, the RGB components are transformed into a gray level image as: $X=0.2 R+0.3 G+0.5 B$.

However, the quality of rock fragment images is affected by different kinds of noise in the image acquisition. Due to the uneven illumination and touching of fragments, the images are not clear enough, which is more difficult for the further image processing. To acquire better image segmentation results, the image preprocessing is necessary because it can reduce the effects, e.g., the smoothing processing aims to weaken the above noise and makes fragment more flat [5]. The improved median filter that makes image smoother (the average gray value of three neighboring gray values) is used in this study and the preprocessing results are shown in Figure 2. 


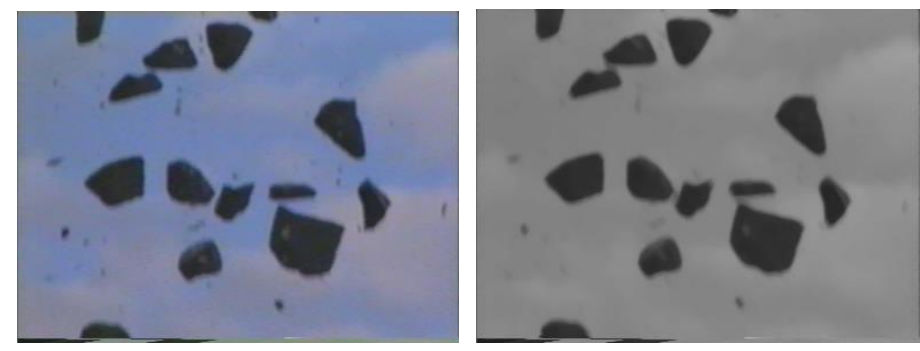

Figure 2. Original Fragment Image and Its Preprocessing Result

\subsection{Initial Thresholding Algorithm}

In the selection of the initial thresholding algorithm, several classical thresholding algorithms have been tested and analyzed. The major tested algorithms contain Otsu, Maximum entropy, Moment-preserving and Iterative. The basic idea of these algorithms is: firstly, to statistic image pixel gray values, then through the grey value histogram to get a gray level threshold $T(0 \leq T \leq 255)$, finally, and to split the entire image into object regions and background regions by $T$. The object regions are where the gray values of pixels are less than $T$, and the background regions are where the gray values of pixels are greater than $T$. The result image can be simply presented as:

$$
f(x, y)=\left\{\begin{array}{cc}
0 & f(x, y) \leq T \\
255 & f(x, y)>T .
\end{array}\right.
$$

The thresholding result for an image intensively relies on $T$, therefore, how to determine $T$ is the key of the image thresholding algorithm. As the comprehensively understanding for the comparison among the algorithms, a brief description for the classical thresholding algorithms (Otsu, Moment-preserving, Maximum entropy, and Iterative) are given as the follows.

The Otsu algorithm was proposed by Otsu in 1979 [6]. The basic idea is assuming that an image can be divided into two classes by using a threshold $T$ : the object class and the background class. Firstly, the numbers of pixels are counted and the average values are calculated for both classes. Subsequently the variance between object and background classes is computed. At last, a gray value $T$ is determined as the threshold when the between-class variance is maximum and the class variance is minimum. It is simple and gets good results for the images with a large grey level difference between objects and background, it meets the real-time request and has been widely used in many vision applications.

The Moment-preserving algorithm was firstly studied by Tsai in 1985 [7]. It is a multi-thresholding algorithm based on image moments; the principle is to remain the moments of an image before and after the operation, and the result is more than one threshold to meet the application requirement. However, the cost of the calculation is too heavy to apply in a real-time system, therefore, in most cases, only one threshold is calculated and adopted, which is suitable for an on-line system.

The Maximum entropy algorithm was suggested by Kapur [8]. The idea is that objects and background in an image are two different types of signal sources, so when the sum of these two types of entropy reaches to the maximum value, the corresponding gray value to distinguish objects from background is the suitable threshold. As seen from the definition of entropy, the smaller the difference is, the larger the entropy is. So if the selected threshold is suitable, the differences among the pixels in different regions can be smaller, the amount of information can be larger and the entropy can be larger; on the contrary, the difference will be larger, the information will be smaller and the entropy will also be smaller. Therefore, when the entropy of object and background regions gets the maximum, the corresponding gray value is the suitable threshold. 
The procedure of the iterative algorithm (or optimal thresholding algorithm) is as follows. Firstly, it is supposed that an image contains two values combined with additive Gaussian noise. Subsequently, on the condition of obtaining the area percentage of objects, the means and their standard deviations can be calculated. Finally, the threshold can be obtained through an optimizing way. The implemented algorithm is for iterative (optimal) threshold selection, which can be found in [9].

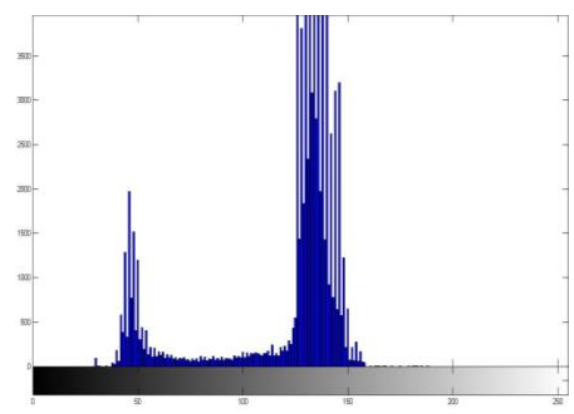

Figure 3. Image Histogram

When choosing a global threshold, there are often two large peaks and one apparent valley in the image histogram, which reflects the difference between the objects and background. The purpose of a thresholding algorithm is to find out the valley as the corresponding threshold. In general, the histogram of a rock fragment image in this study has a valley more or less (Figure 3). While the thresholds obtained by using the above described algorithms are different, they all fall in the valley region, so the segmentation results are different but similar.

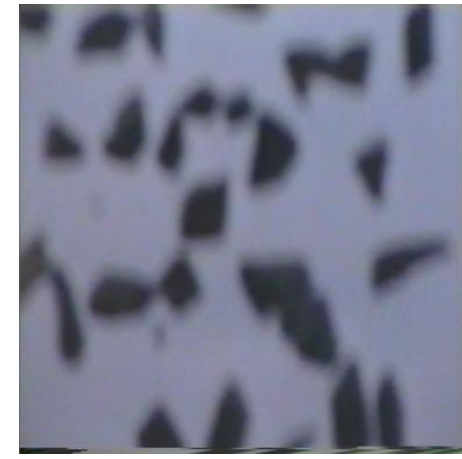

(a) Original image

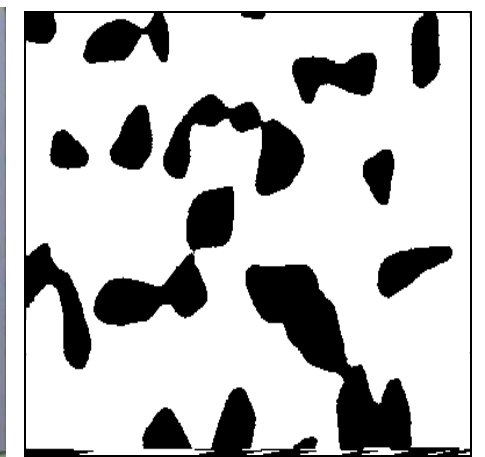

(b) Otsu result

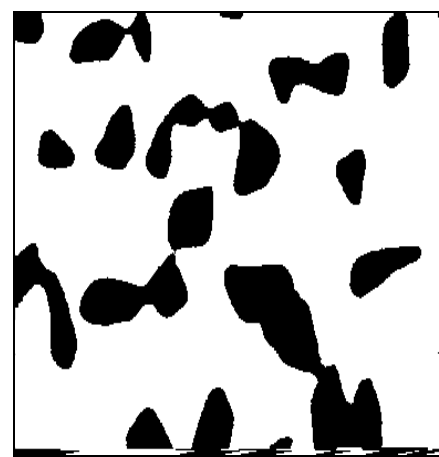

(c) Iteration result

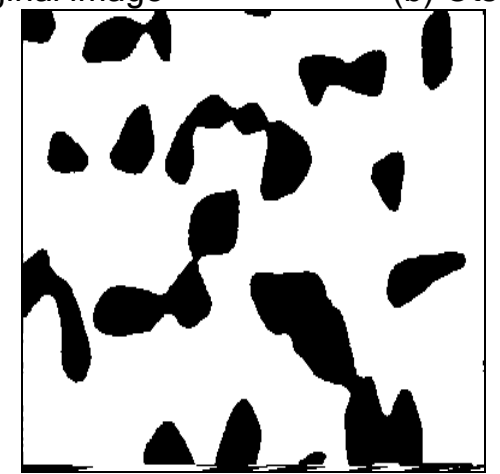

(d) Moment-preserving result

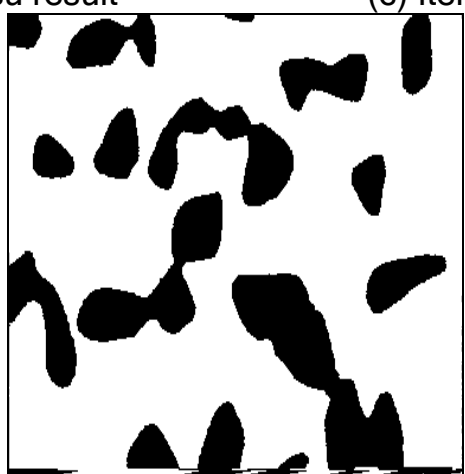

(e) Maximum entropy result

Figure 4. Results of Four Widely Used Thresholding Algorithms

The experiment is carried out for comparing the global thresholding algorithms, and a 
number of the falling rock fragment images (No.0-300) are used. As Figure 4 and Table 1 shown, the Moment-preserving and Maximum entropy algorithms give a larger $T$ which outputs the larger fragments than real ones, while the Otsu and Iteration result in a smaller $T$ which produces a better result. However, the Iteration result depends on the pre-set initial threshold for some images, sometimes, the wrong threshold is obtained, e.g. $T=0$. Hence the Otsu algorithm is selected in this study. It can be seen from Figure 4, after the initial segmentation, the fragments are overlapping due to the small differences. Therefore, for these overlapping regions, a smart split algorithm is studied based on a number of rules.

Table 1. Results of Four Threshold Segmentation Algorithms

\begin{tabular}{|l|l|l|l|l|}
\hline Image number & OTSU & Moment preserving & Iteration & Max entropy \\
\hline 0 & 102 & 104 & 102 & 117 \\
\hline 1 & 103 & 104 & 103 & 111 \\
\hline 3 & 99 & 101 & 100 & 111 \\
\hline 38 & 97 & 103 & 96 & 121 \\
\hline 44 & 100 & 105 & 100 & 119 \\
\hline 116 & 100 & 105 & 100 & 121 \\
\hline 118 & 100 & 104 & 101 & 111 \\
\hline 120 & 102 & 104 & 102 & 111 \\
\hline 130 & 103 & 108 & 103 & 121 \\
\hline 150 & 103 & 105 & 103 & 111 \\
\hline 152 & 103 & 105 & 102 & 117 \\
\hline 157 & 104 & 105 & 104 & 111 \\
\hline 159 & 101 & 102 & 101 & 111 \\
\hline 177 & 101 & 105 & 100 & 121 \\
\hline 182 & 103 & 109 & 103 & 121 \\
\hline 183 & 102 & 108 & 102 & 121 \\
\hline 184 & 104 & 109 & 104 & 125 \\
\hline 193 & 103 & 107 & 103 & 117 \\
\hline 228 & 103 & 106 & 103 & 111 \\
\hline 238 & 102 & 105 & 102 & 111 \\
\hline 255 & 103 & 106 & 103 & 111 \\
\hline & & & & \\
\hline
\end{tabular}

\section{Image Segmentation based on Fuzzy Math}

The further image segmentation is to split touching fragments in a binary image which is the result from the initial global thresholding algorithm. To solve the fragment overlapping problem, the touching regions (clusters) are detected based on different rules, then, the Otsu operation is carried out on the cluster regions. The two steps are repeated until there is no region to be split.

\subsection{Definitions of Parameters for a Cluster Region}

As the literatures review, it can be faster and easier to split cluster regions of the rock fragments based on the size and shape characteristics in some cases [9-12], but it can inevitably produce the fault split lines without using the grey level information. Although the gray level difference among the rock fragments in a touching region is relatively small, the gradient and smoothness information of the touching region can be the basis for separating the clusters. The information of the gradient magnitude and gray level smoothness can still be extracted, so it can be used to describe the changes of the edges and the gray levels inside the region. As analyzed, the following characteristics can be used to identify whether a region is a fragment touching cluster or not.

As the rock fragments are produced for railway, the size of fragments should be between $32-64 \mathrm{~mm}$, and the shape of the fragments is usually an irregular polygon. So the major description about size and shape of an object region is as follows: 
1) Area of a region: $S$

It can be seen from the result of the global threshold segmentation that a very large area is the most significant feature of overlapping fragments. The area of a region can be defined with the number of pixels. The stable and simple way to calculate the area is to count the number of pixels within and on the boundary [13-14]. If the area of a region is so large, far greater than the threshold, i.e. $S \gg>T_{S}$, the region should be a fragment overlapping cluster.

2) Perimeter of a region: $L$

The perimeter of a region is important in distinguishing simple or complex shaped objects by combining the area. The perimeter of a region is defined as the length of boundary which belongs to the region. There are different representations to describe the perimeter, the calculation methods are different. In this paper, the perimeter is defined as the boundary area. If the perimeter of a region is very long, far greater than a threshold, i.e. $L>>T_{L}$, the region should be a cluster.

3) Shape factor of a region: $F$

The shape factor here is used for describing the approach extent of a region to a circular, the shape factor of a circular is 1 , so, the larger the difference between an object and a circular in shape is, the greater the difference between the object shape factor and 1 is [15-16]. It defined as:

$$
F=\frac{L^{2}}{4 \pi S}
$$

4) Gradient magnitude of an internal region: $G$

After edge detection, the larger gradient magnitudes might show that the region is formed by more than one fragment. Gradient magnitudes can effectively show the variation of gray values in a region. The differential value of a random pixel in an image can indicate the change rate of its gray value, so it is used to define the gradient magnitudes, it sets the image function as $f(x, y)$, and the definition of gradient magnitudes at a point $(x, y)$ is as follows:

$$
G[f(x, y)]=\left[(\partial f / \partial x)^{2}+(\partial f / \partial y)^{2}\right]^{1 / 2}
$$

Generally it uses the difference to approximate the differential; a typical formula can be defined as:

$$
G[f(x, y)] \approx\left\{[f(x, y)-(x+1, y)]^{2}+[f(x, y)-f(x, y+1)]^{2}\right\}^{1 / 2}
$$

5) Smoothness of an internal region: $R$

The texture information is a basic characteristic in an image and the smoothness is its corresponding parameter; if a region is not smooth, it might be a cluster. The gray value variance of an image region can reflect the smoothness. It is defined as:

$$
R=\frac{1}{M N} \sum_{i=1}^{M} \sum_{j=1}^{N}\left(I(i, j)-\bar{I}\left(i_{0}, j_{0}\right)\right)^{2}
$$

Where, $M \times N$ is the window size of a selected region, $\bar{I}\left(i_{0}, j_{0}\right)$ is the average gray value of the region.

\subsection{Fuzzy Comprehensive Evaluation for Rock Fragment Parameters}

There are many factors to be considered when considering the rock fragment parameter information as the basis for the further thresholding operation. If only one factor is considered, the accurate judgment cannot be got. In addition, it is usually not manifest whether every object region needs to be further divided or not. So the fuzzy 
comprehensive evaluation method, by integrating the evaluations of the major factors for each region, is used for solving this problem. The fuzzy comprehensive evaluation uses the principle of fuzzy relation synthesis, selects some factors that are ambiguously defined or difficult to do quantitative analysis, and makes the comprehensive evaluation. The main idea of the fuzzy comprehensive evaluation is to use the fuzzy linear transform principle and the maximum degree of membership principle, considering various factors related to the evaluation and making a reasonable comprehensive evaluation [17]. The comprehensive evaluation involves three elements:

(1) Factor set: $U=\left\{u_{1}, u_{2}, \cdots, u_{m}\right\}$;

(2) Comment set: $V=\left\{v_{1}, v_{2}, \cdots, v_{n}\right\}$;

(3) Single factor fuzzy evaluation, namely to evaluate the single factor $u_{i}(i=1, \cdots, m)$ : get a set $F\left(r_{i 1}, r_{i 2}, \cdots, r_{i n}\right)$ belonging to $V$, so it is a $F$ mapping from $U$ to $V$, the $F$ mapping can determine a $F$ relationship $R \in \mu_{m \times n}, R$ is the evaluation matrix:

$$
R=\left[\begin{array}{cccc}
r_{11} & r_{12} & \cdots & r_{1 n} \\
r_{21} & r_{22} & \cdots & r_{2 n} \\
\vdots & \vdots & & \vdots \\
r_{m 1} & r_{m 2} & \cdots & r_{m n}
\end{array}\right]
$$

While each of the evaluation factors has a different weight to make the decision on the evaluation result, it is required to weigh every evaluation factor. $A=\left(a_{1}, a_{2}, \cdots, a_{m}\right)$ indicates the weights of the factors. By combining the weight set and the evaluation matrix, the comprehensive evaluation set is obtained as $B=\left(b_{1}, b_{2}, \cdots, b_{n}\right)$. Finally, based on the principle of the maximum degree, the biggest factor selected from the comprehensive evaluation set is the comprehensive evaluation result.

The factor set consisting of the rock fragment parameters is the basis of the comprehensive evaluation and it will affect the authenticity, reliability of the evaluation result. The selected factors should be able to fully reflect the gray scales of fragments and shape features clearly. So, area, perimeter, shape factor, gradient magnitudes and smoothness of an object region are selected to be the main factors as a set. The result of the comprehensive evaluation, namely the comment set, can give the information if the detecting region needs to be further split. There are many methods to determine the weight of each factor; the widely used method is the statistical one, such as Analysis Hierarchy Process. The statistical method requires to query the professional experts who have rich experience to give the most appropriate weight of each factor and to make statistical analysis.

For the definition of the evaluation matrix, the effect for the evaluation result caused by each of the factors individually is to be known. the evaluation matrix is made up with the membership degree of each factor, therefore, the determination of the membership is the key step. There are a variety of ways to determine the membership function, such as rationalistic, fuzzy statistics, tracheotomy, dualistic contrast compositor method and so on. Fuzzy statistics method requires a lot of statistical experiments to determine the result by using the logical reasoning. For example, to determine the membership of a fixed element $u_{0}$ belonging to universal $U$ to a fuzzy set $A^{*}, u_{0}$ is fixed, and $A^{*}$ is a dynamic set. After $n$ times fuzzy statistical experiments, one can determine the degree of the membership like this:

$$
\text { the membership } u_{0} t o A=\frac{\text { times of } " u_{0} \in A "}{n}
$$

With the increase of $n$, the frequency of the membership is of stability, which is the membership degree of $u_{0}$ to $A$. After many statistics for rock fragmentation images and 
observation of the touching fragment situation, the membership of each factor in the region can be obtained, thus, the curve fit is made and the membership function is got. After statistics, the membership of each factor is stable and these memberships form the member functions as follows: (the function fitting results are shown in Figure 5)

The membership function of the area (see Figure 5) and the evaluation parameters of the fitting result are: SSE: 0.75 , R-square: 0.9823

$$
\begin{aligned}
F(x) & =0.008131 x^{9}-0.03741 x^{8}-0.001882 x^{7}+0.2197 x^{6}-0.2257 x^{5}-0.303 x^{4}+0.5725 x^{3}-0.2227 x^{2} \\
& -0.01311 x+1.008
\end{aligned}
$$

The membership function of the perimeter (see Figure 5) and the evaluation parameters of the fitting result are: SSE: 2.007, R-square: 0.9361

$$
\begin{aligned}
F(x) & =0.007753 x^{9}-0.03216 x^{8}+0.2362 x^{7}-0.1396 x^{6}-0.4537 x 5+0.5381 x^{4}-0.04068 x^{3} \\
& -0.09318 x+1.01
\end{aligned}
$$

The membership function of the shape factor (see Figure 5) and the evaluation parameters of the fitting result are: SSE: 0.0473, R-square: 0.9601

$$
\begin{aligned}
F(x) & =0.006222 x+0.4685 \quad(x<50) ; \\
F(x) & =-0.02712 x^{4}+0.1385 x^{3}-0.1587 x^{2}-0.1711 x+0.7113 \quad(50 \leq x \leq 275) ; \\
F(x) & =39.63 x^{9}-91.06 x^{8}-77.39 x^{7}+193.1 x^{6}+88.61 x^{5}-111.6 x^{4}-48.56 x^{3}+12.94 x^{2}+6.111 x \\
& +0.8071(x>275) .
\end{aligned}
$$

The membership function of gradient magnitudes (see Figure 5) and the evaluation parameters of the fitting result are: SSE: $0.6665, \mathrm{R}$-square: 0.8756

$$
\begin{aligned}
& F(x)=0.3408 x+0.2591 \quad(x<30) \text {; } \\
& F(x)=0.02721 x^{9}-0.1422 x^{8}+0.1277 x^{7} 0.398 x^{6}-0.65481 x^{5}-0.1142 x^{4}+0.4862 x^{3}-0.07631 x^{2} \\
& -0.06878 x+0.1478 \quad(30 \leq x \leq 208) ; \\
& F(x)=0.1546 x^{4}-0.2838 x^{3}-0.2729 x^{2}+0.7019 x+0.7214 \quad(x>208) .
\end{aligned}
$$

The membership function of the smoothness (see Figure 5) and the evaluation parameters of the fitting result are: SSE: 1.145 , R-square: 0.8072

$$
\begin{aligned}
& F(x)=0.3532 x+0.3337 \quad(x<68) ; \\
& F(x)=0.1113 x^{4}-0.05123 x^{3}-0.4865 x^{2}-0.1153 x+0.6175 \quad(68 \leq x \leq 200) ; \\
& F(x)=0.1238 x^{2}-0.07593 x+0.2968 \quad(x>200) .
\end{aligned}
$$

For the fitting function, the original data has to be normalized and centered, the centered method is:

$$
y=x-\operatorname{mean}(x) / \operatorname{std}(x)
$$

Where, $\mathrm{x}$ is the original data, mean is the mean of $\mathrm{x}$, and std is the standard deviation of $\mathrm{x}$. Accordingly, the above expression in function $\mathrm{x}$ is a data conducted through the center and normalization. The standard of the function partition is with the normalized data. There are errors between fitting and experimental data, the parameters to evaluate the result are: the sum of squares due to error (SSE) and the determination coefficient (R-square).

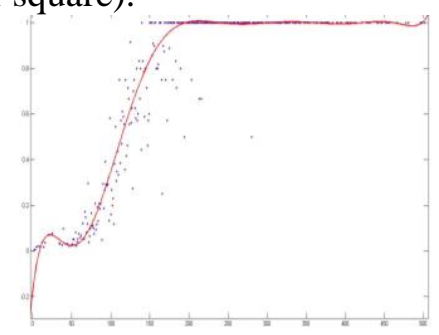

(a) Membership function of $S$

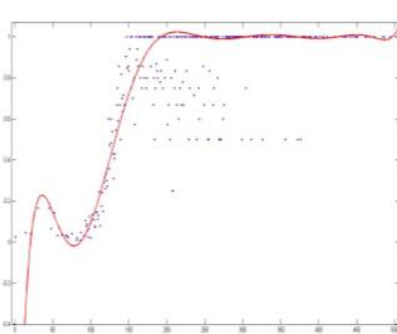

(b) Membership function of

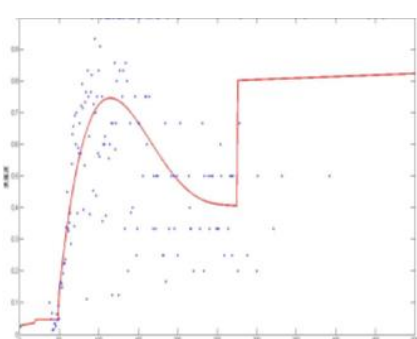

(c) Membership function of $F$ 


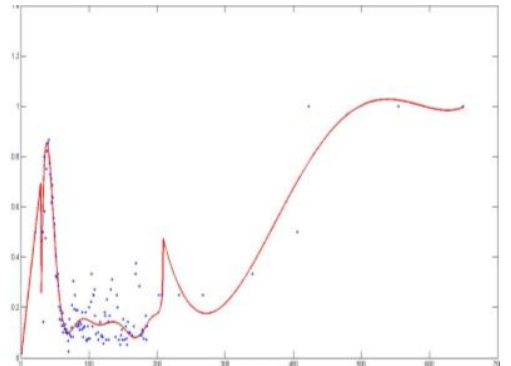

(d) Membership function of $G$

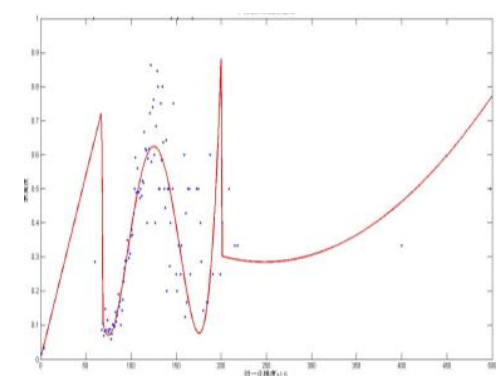

(e) Membership function of $R$

Figure 5. Five Membership Functions for Rock Fragments

For evaluating the curve fitting, SSE is closed to 0 , the better result of the curve fitting is obtained and R-square is closed to 1, the better result of the curve fitting is acquired. Each quality parameter of the curve fitting is shown above, and the result is well (see Figure 5).

For each fragment region, after getting its parameters, the evaluation matrix is obtained by the membership functions. Then the evaluation matrix is combined with the weight set to get the final comprehensive evaluation set. The weight set derived by statistical analysis is: $\mathrm{A}=(0.4,0.2,0.2,0.1,0.1)$.

Each of the factors is corresponding to area, perimeter, shape factor, gradient magnitudes, and smoothness.

\section{Experiments and Comparison Analysis}

\subsection{Fuzzy Comprehensive Evaluation Results}

The parameter information of the initial image segmentation result needs to be acquired before the fuzzy comprehensive evaluation. The multiple images are analyzed, as an example, after labeling and tracking for each object, the area, perimeter and shape factors are obtained. These parameters such as gradient magnitudes and smoothness data of the original image are shown in Table 2.

Table 2. Parameters Obtained from Otsu Algorithm

\begin{tabular}{|l|l|l|l|l|}
\hline $\begin{array}{l}\text { Area } \\
\text { (pixel) }\end{array}$ & $\begin{array}{l}\text { Perimeter } \\
\text { (pixel) }\end{array}$ & Shape factor & Gradient & Smoothness \\
\hline 80 & 45 & 2.0143 & 5.2773 & 92.2953 \\
\hline 210 & 123 & 5.733 & 13.1659 & 461.9497 \\
\hline 521 & 84 & 1.0777 & 4.1948 & 166.4494 \\
\hline 653 & 84 & 0.8599 & 7.7726 & 118.5187 \\
\hline 721 & 93 & 0.9546 & 6.3419 & 127.6846 \\
\hline 790 & 105 & 1.1106 & 8.559 & 115.6598 \\
\hline 1056 & 119 & 1.0671 & 6.0859 & 115.0897 \\
\hline 1091 & 112 & 0.915 & 13.061 & 126.4722 \\
\hline 1265 & 148 & 1.3779 & 4.5952 & 152.8139 \\
\hline 1543 & 312 & 5.0203 & 4.9893 & 247.6088 \\
\hline 1560 & 194 & 1.9199 & 5.1976 & 140.3962 \\
\hline 1638 & 183 & 1.627 & 5.3799 & 137.1234 \\
\hline 2028 & 241 & 2.2791 & 3.5323 & 176.308 \\
\hline 3242 & 349 & 2.9897 & 4.6111 & 192.1662 \\
\hline
\end{tabular}




\begin{tabular}{|l|l|l|l|l|}
\hline 4055 & 358 & 2.5152 & 3.2041 & 195.8393 \\
\hline 6844 & 607 & 4.2841 & 3.6239 & 217.3988 \\
\hline
\end{tabular}

In Figure 4, for example, the objects are labeled in the binary image (Figure 4(b)) after the grey level image segmentation by using a global threshold, the boundary chain code method is adopted to gain area, perimeter and shape factor of each rock fragment region. Gradient magnitudes and smoothness information are obtained from the original image regions (corresponding to the labeled regions in the binary image). The parameters obtained from the initial thresholding algorithm are firstly normalized and centered, and then the membership of each individual factor through the membership function is calculated, next, the membership is combined into the evaluation matrix, finally, the evaluation matrix is multiplied to the weight set to gain the final evaluation set. The two sets of rock fragment parameters are taken from Table 2, e.g., the fuzzy comprehensive evaluation is performed. The main working steps are as follows:

According to the principle of the maximum membership degree, the regions 1 and 2 do not need the further image segmentation, but the region 3 needs to be further divided.

1) Factor set:

Table 3. First Set of Data

\begin{tabular}{|l|l|l|l|}
\hline & Original Data & Centered Data & Membership \\
\hline Area & 210 & -1.7023 & 0.05292 \\
\hline Perimeter & 123 & -1.2035 & 0.1492 \\
\hline Shape Factor & 5.733 & 2.1622 & 0.4067 \\
\hline Gradient & 13.1659 & 0.7157 & 0.1421 \\
\hline Smoothness & 461.9497 & 0.1480 & 0.2883 \\
\hline
\end{tabular}

Table 4. Second Set of Data

\begin{tabular}{|l|l|l|l|}
\hline & Original Data & Centered Data & Membership \\
\hline Area & 1056 & -1.2273 & 0.0861 \\
\hline Perimeter & 119 & -1.2407 & 0.1068 \\
\hline Shape Factor & 1.0671 & -1.3352 & 0.2410 \\
\hline Gradient & 6.0859 & -0.8335 & 0.1382 \\
\hline Smoothness & 115.0897 & -1.2965 & 0.3753 \\
\hline
\end{tabular}

Table 5. Third Set of Data

\begin{tabular}{|l|l|l|l|}
\hline & Original Data & Centered Data & Membership \\
\hline Area & 4055 & 0.469 & 0.9944 \\
\hline Perimeter & 358 & 0.6087 & 0.9964 \\
\hline Shape Factor & 2.5152 & -0.1585 & 0.7339 \\
\hline Gradient & 3.2041 & -1.4444 & 0.7430 \\
\hline Smoothness & 195.8393 & 0.3092 & 0.5348 \\
\hline
\end{tabular}

2) Evaluation matrix:

3) Weight set:

$$
R_{1}=\left[\begin{array}{ll}
0.0529 & 0.9471 \\
0.1492 & 0.8508 \\
0.4067 & 0.5933 \\
0.1421 & 0.8579 \\
0.2883 & 0.7117
\end{array}\right] \quad R_{2}=\left[\begin{array}{ll}
0.0861 & 0.9139 \\
0.1068 & 0.8932 \\
0.2410 & 0.7590 \\
0.1382 & 0.8618 \\
0.3753 & 0.6247
\end{array}\right] \quad R_{3}=\left[\begin{array}{ll}
0.9944 & 0.0056 \\
0.9964 & 0.0036 \\
0.7339 & 0.2661 \\
0.7430 & 0.2570 \\
0.5348 & 0.4652
\end{array}\right]
$$

$\mathrm{A}=(0.4,0.2,0.2,0.1,0.1)$

4) Comprehensive evaluation set: 
$\mathrm{B} 1=\mathrm{A} * \mathrm{R} 1=(0.17010 .7299)$

$\mathrm{B} 2=\mathrm{A} * \mathrm{R} 2=(0.14670 .7533)$

$\mathrm{B} 3=\mathrm{A} * \mathrm{R} 3=(0.77220 .1278)$

\subsection{Final Image Segmentation Result Analysis}

The local threshold segmentation is carried out on the basis of the results by the initial global thresholding algorithm. The result of the initial segmentation will have an effect on the result of the local thresholding, thus, only the local thresholding results in the same case with the global thresholding are compared. The final result of the adaptive thresholding algorithm is shown in Figure 7 and Figure 8.

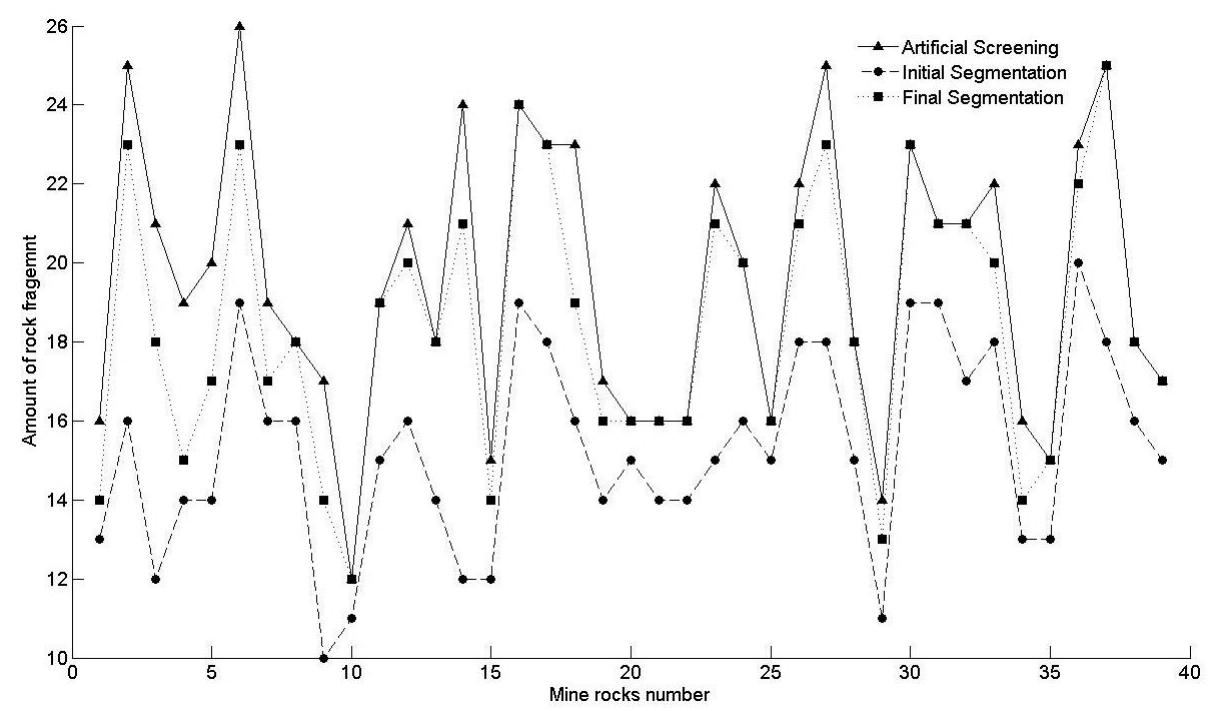

Figure 6. Artificial Screening, Initial and Final Segmentation Results

There is little difference among the fragment numbers of sieving, initial segmentation and new segmentation algorithms for the same image. A number of images are chosen to process by using these methods and the fragment numbers from the three methods are shown in Figure 6. Compared to the initial segmentation, the result from the new segmentation algorithm is close to the one by using the sieving method, and its accuracy rate is up to $90 \%$. Touching regions are well separated on the basis of the initial segmentation, and the final image segmentation results for different images by the new algorithm are shown in Figure 7. It can be seen that after the initial segmentation, there are shadows in fragments (as arrow illustrated), they can be separated by the local thresholding algorithm especially for the shadow part.

In Figure 7(a1), there is a large cluster in the middle of the image, it includes at least 7 fragments as shown in (b1), 7 touching fragments are well split based on gradient, shape and grey level flatness, but it is difficult to adjust if the remaining relative large object is a touching cluster. The similar situation is also in Figure 7(a2-c2). In Figure 7(a3, a4), there are several clusters in each image, but the largest cluster in an image involves only 3 fragments, the segmentation results are satisfactory. Table 6 shows the detailed segmentation information and the processing accuracy is over 95\% compared to human vision. We did the same tests for more than 300 images, the results are similar. Even though, there might be some special touching regions that cannot be split by the studied algorithm, which might be caused by other reasons such as fuzzy shapes and very vague touching regions, which is the further work for the continuation of this studies.

Another example is shown in Figure 8 and Table 7 and the results in each step by the new algorithm are presented. In some original images, there are some fragment shadows. 
It will affect the image segmentation results by an ordinary image segmentation algorithm while through the new algorithm the influence can be intensively decreased. As Table 7 shown, the accuracy is over $96 \%$.

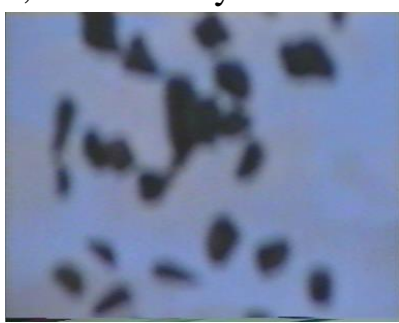

(a1)

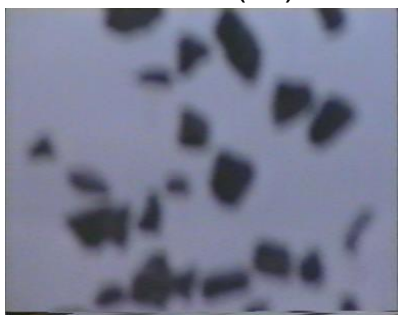

(a2)

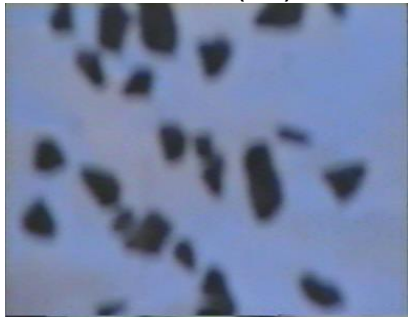

(a3)

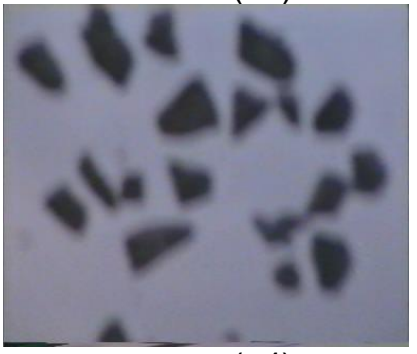

(a4)

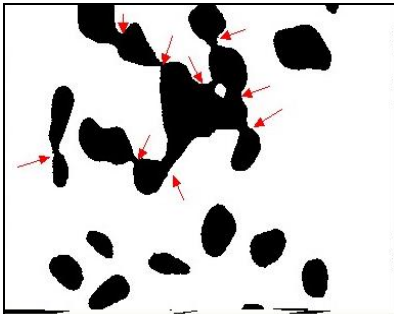

(b1)

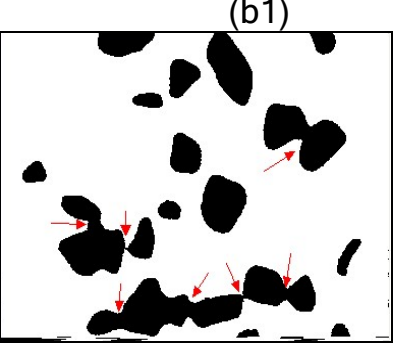

(b2)

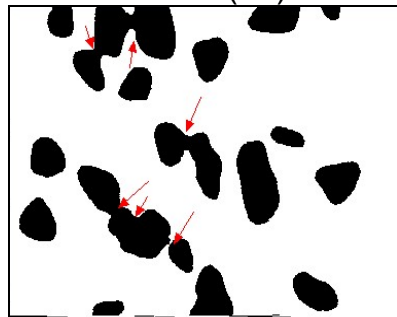

(b3)

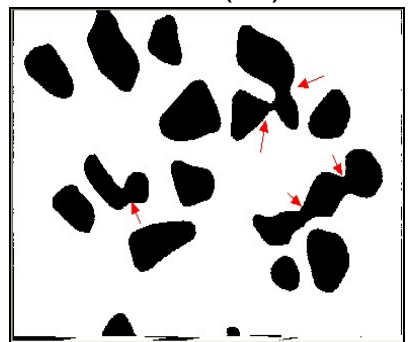

(b4)
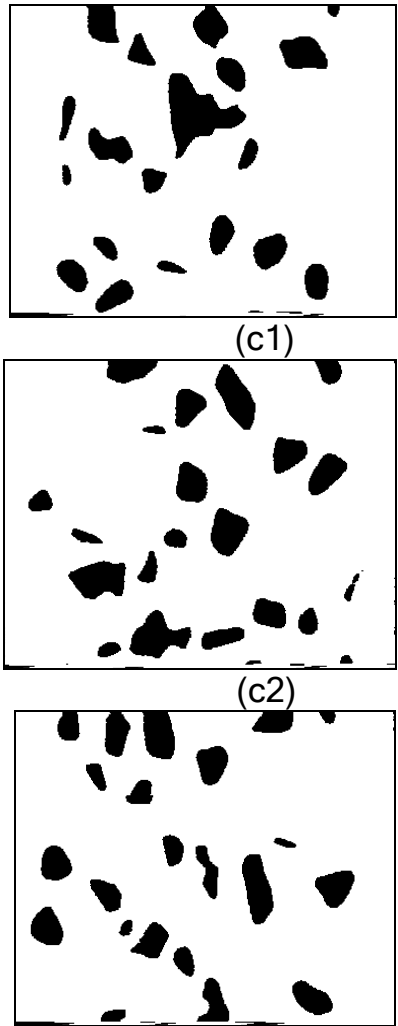

(c3)

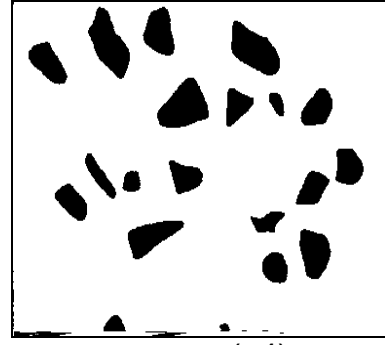

(c4)

Figure 7. Initial and Final Image Segmentation Results: (A) Original Image;

(B) Initial Segmentation Results; and (C) Final Segmentation Results

Table 6. Image Segmentation Result Analysis For Figure 7

\begin{tabular}{|c|c|c|c|c|}
\hline Original image & Fragment No. & Cluster No. & Result No. & Accuracy \\
\hline $\mathrm{a} 1$ & 23 & 2 & 22 & $95.7 \%$ \\
\hline $\mathrm{a} 2$ & 26 & 4 & 25 & $96.2 \%$ \\
\hline $\mathrm{a} 3$ & 26 & 3 & 25 & $96.2 \%$ \\
\hline $\mathrm{a} 4$ & 23 & 3 & 22 & $95.7 \%$ \\
\hline Sum & 98 & 12 & 94 & $95.9 \%$ \\
\hline
\end{tabular}




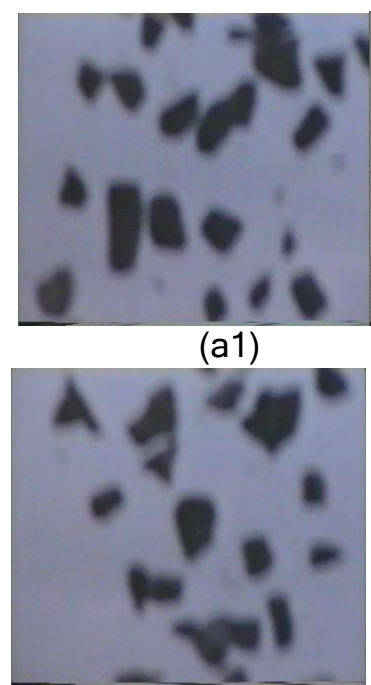

(a2)

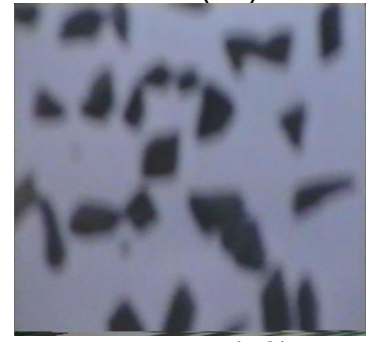

(a3)

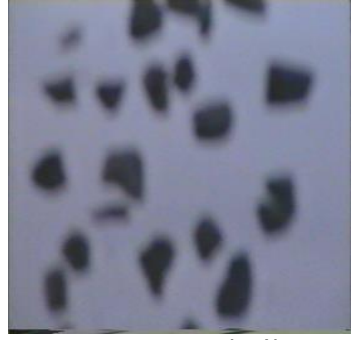

(a4)

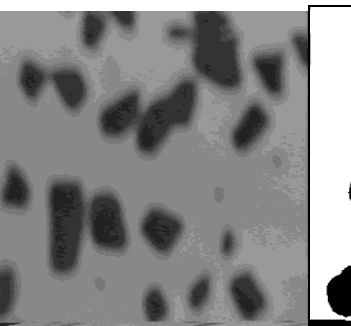

(b1)

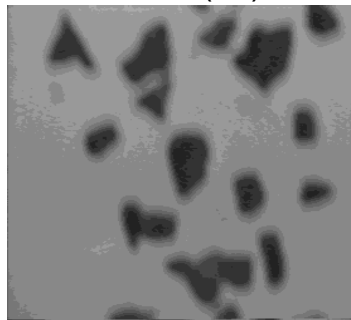

(b2)

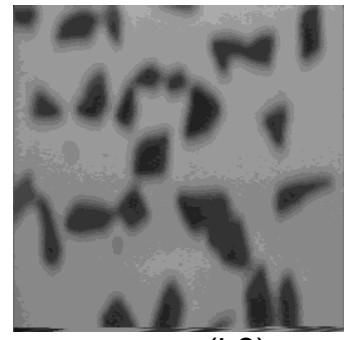

(b3)

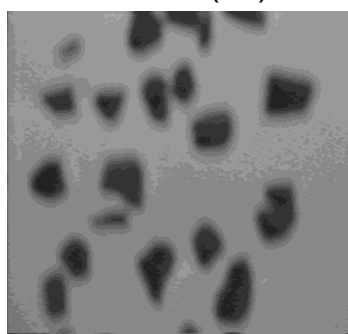

(b4)

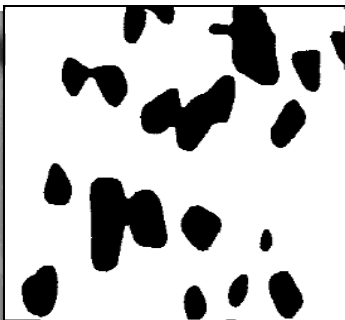

(c1)

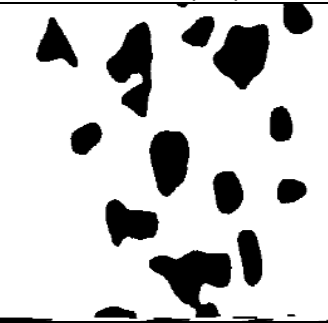

(c2)

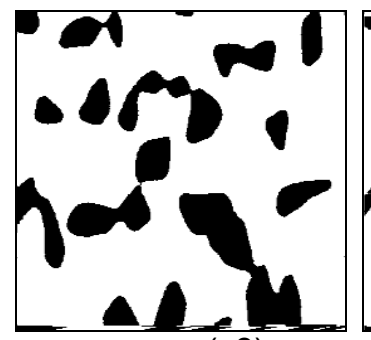

(c3)

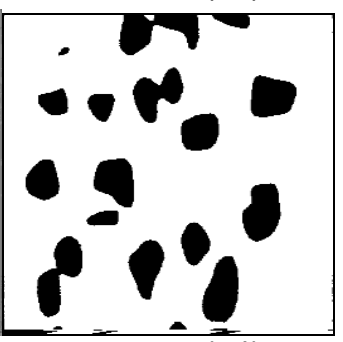

(c4)

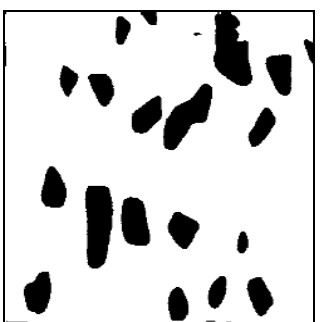

(d1)

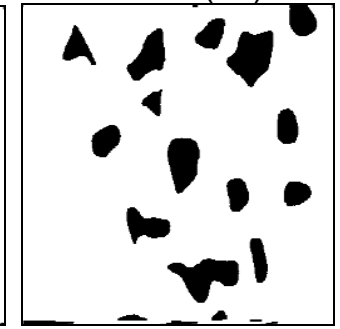

(d2)

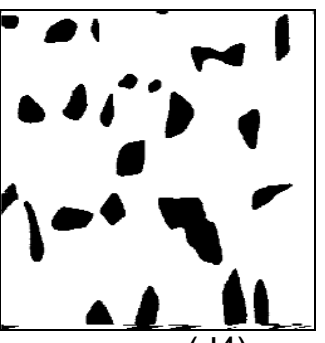

(d4)

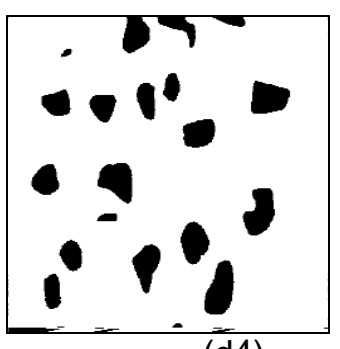

Figure 8. Results Of Each Step In The New Algorithm: (A) Original Color Images; (B) Gray Scale Images; (C) Initial Segmentation; And (D) Final

Segmentation

Table 7. Image Segmentation Result Analysis for Figure 8

\begin{tabular}{|c|c|c|c|c|}
\hline Original image & Fragment No. & Cluster No. & Result No. & Accuracy \\
\hline a1 & 20 & 5 & 20 & $100 \%$ \\
\hline a2 & 20 & 2 & 20 & $100 \%$ \\
\hline a3 & 28 & 7 & 27 & $96.4 \%$ \\
\hline a4 & 22 & 3 & 22 & $100 \%$ \\
\hline Sum & 90 & 17 & 89 & $98.9 \%$ \\
\hline
\end{tabular}

\subsection{Comparison to Other Algorithms}

To validate the new image segmentation, other three classical image segmentation algorithms (Cluster analysis, Graph based, FCM) [18-20] are coded and used to test the 100 fragment images in this study. Figure 9 and Table 8 show an example of the comparison of four algorithms. For the image in (a1), the Cluster analysis algorithm and the Graph based algorithm merge the fragments at the right top and background into a large cluster, the FCM algorithm outputs edge regions with clusters, the new algorithm gives a 
excellent segmentation result where the fragments cut by image frame are of light color and the split lines are also in the light color. For the image in (a2), the three classical algorithms produce a lot of clusters (5-8), in addition, the Cluster analysis algorithm outputs only the edge regions without fragments in some parts, which is different to the FCM algorithm that gives the edge region for every fragment, the new algorithm performs relatively well, except for one cluster which is vague by human vision. The other two image processing results are the similar to that above.

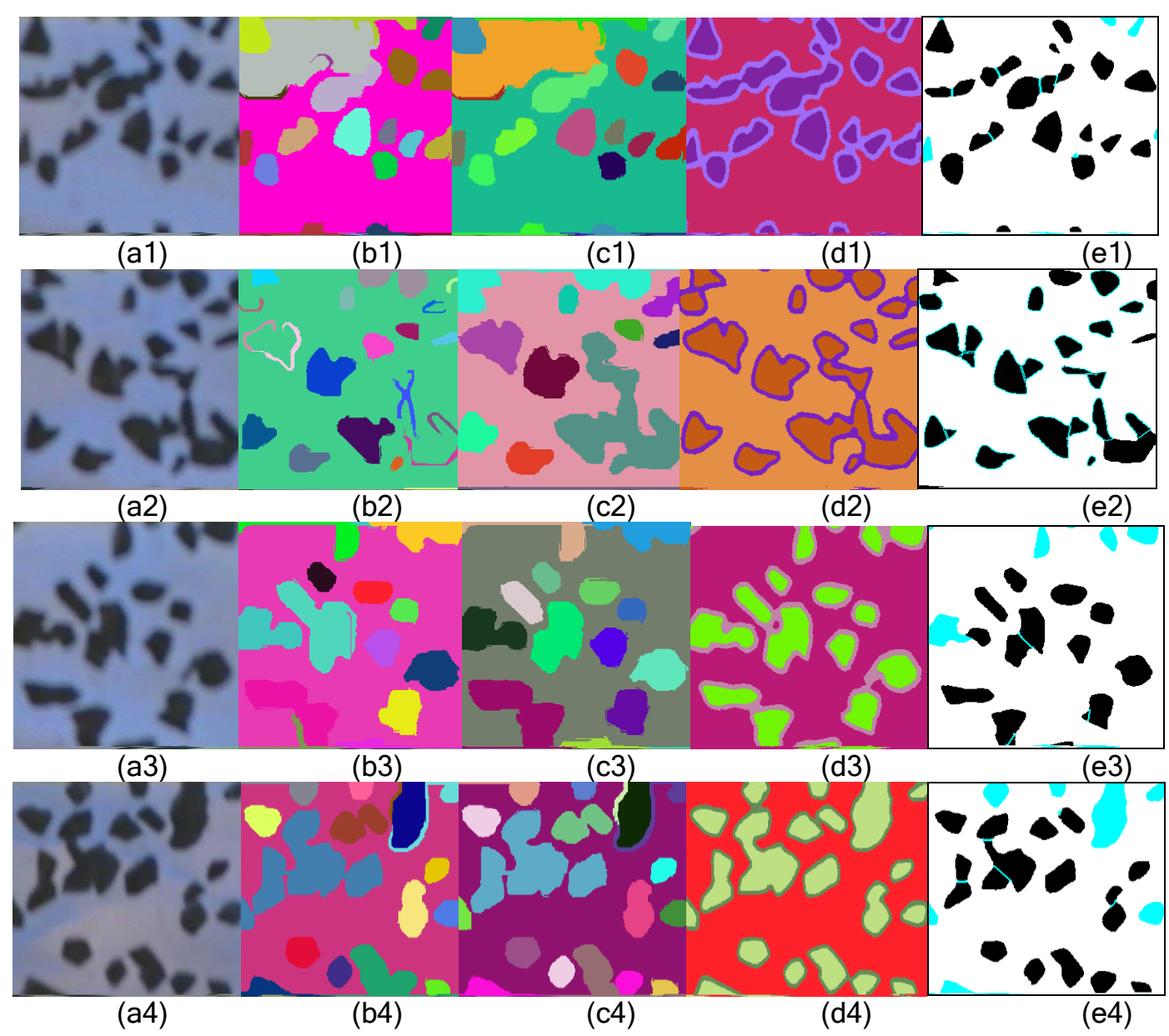

Figure 9. Image Segmentation Results By Using New Algorithm And Other Three Algorithms: (A) Original Image; (B) Clustering Analysis; (C) Graph Segmentation; (D) Fcm Segmentation; And (E) New Algorithm

Table 8. Comparison Between Four Algorithms For Figure 9

\begin{tabular}{|c|c|c|c|c|}
\hline Original image & Clustering analysis & $\begin{array}{c}\text { Graph } \\
\text { segmentation }\end{array}$ & FCM segmentation & New algorithm \\
\hline $\mathrm{a} 1$ & 2 & 3 & 3 & 0 \\
\hline $\mathrm{a} 2$ & 8 & 7 & 5 & 1 \\
\hline $\mathrm{a} 3$ & 6 & 6 & 3 & 1 \\
\hline $\mathrm{a} 4$ & 4 & 4 & 3 & 1 \\
\hline Sum & 20 & 20 & 14 & 3 \\
\hline
\end{tabular}

\section{Conclusions}

Image segmentation is the key technology for rock fragments in an on-line system, compared to the widely used classical algorithms (Cluster analysis, Graph based, FCM), the general global thresholding algorithm is simple, fast and stable. However, it is difficult 
to solve the fragment overlapping problem. This paper studied an adaptive thresholding algorithm which is on the basis of image preprocessing by using the Otsu thresholding algorithm for the initial image segmentation, after that, based on the fragment shape, size, gradient magnitudes and gray level information, it takes advantage of rock fragment characteristic parameters to find out the touching fragment clusters for the further splitting by using fuzzy comprehensive evaluation method. In the fuzzy comprehensive evaluation method, the area, perimeter, shape factor, gradient magnitudes and smoothness for an object region are selected to setup the different membership functions that are defined by a large number of tests in this study. The experimental results show that the algorithm can make a better effect for rock fragment image segmentation than the Cluster analysis, the Graph based and the FCM algorithms; therefore, it can be applied for the rock fragment detection in an on-line system of falling stream fragments. The further research is to expand the applications of the new algorithm, such as other on-line detection/monitoring systems which can acquisite the similar images containing complex and touched multi-objects (e.g., various particle images).

\section{References}

[1] A. K. Raina, "A History of Digital Image Analysis Technique for Blast Fragmentation Assessment and Some Indian Contributions", Edited Sanchidrián \& Singh, Taylor \& Francis Group, London, (2013), pp. 3-11.

[2] H. Zhang, "Image Processing for the Oil Sands Mining Industry, IEEE Signal Processing Magazine, vol. 6 , no. $25,(\mathbf{2 0 0 8})$, pp. $197-200$.

[3] M. J. Thurley, "Automated Online Measurement of Limestone Particle Size Distributions Using 3D Range Data", Journal of Process Control, vol. 2, no. 21, (2011), pp. 254-262.

[4] E. Hamzeloo, M. Masinaei and N. Mehrshad, "Estimation of Particle Size Distribution on a Conveyor Belt Using Image Analysis and Neural Network", International Mineral Processing Congress, (2012).

[5] F. I. Siddiqui, S. M. A. Shah and M. Y. Behan, "Measurement of Size Distribution of Blasted Rock Using Digital Image Processing”, JKAU: Eng. Sci., vol. 2, no. 20, (2009), pp. 81-93.

[6] N. Otsu, "A Threshold Selection Method from Gray-level Histograms", IEEE Trans. System Man Cybernetics, vol. 1, no. 9, (1979), pp. 62-66.

[7] W. H. Tsai, "Moment-preserving Thresholding: a New Approach", Computer Vision Graphics Image Process, vol. 3, no. 29, (1985), pp. 377-393.

[8] J. N. Kapur, P. K. Sahoo and A. K. C. Wong, "A New Method for Gray-level Picture Thresholding Using the Entropy of the Histogram”, Computer Vision, Graphics, and Image Processing, vol. 3, no. 29, (1985), pp. 273-285.

[9] M. Sonka, V. Hlavac and R. Boyle, Image Processing, Analysis and Machine Vision, Champion \& Hall, London, (1995).

[10] Z. L. Zhang, J. G. Yang, X. L. Su and L. H. Ding, "Analysis of Large Particle Sizes Using a Machine Vision System”, Physicochem. Probl. Miner. Process, vol. 2, no. 49, (2013), pp. 397-405.

[11] I. Kinnunen and A. Makynen, "Image Based Size Distribution Measurement of Gravel Particles", Instrumentation and Measurement Technology Conference, (2011).

[12] N. H. Maerz, "Technical and Computational Aspects of the Measurement of Aggregate Shape by Digital Image Analysis", Journal of Computing in Civil Engineering, vol. 1, no. 18, (2004), pp. 10-18.

[13] W. X. Wang, "Image Analysis of Particles by Modified Ferret Method-best-fit Rectangle", Powder Technology, vol. 1, no. 165, (2006), pp. 1-10.

[14] M. J. Thurley, "Automated, On-line, Calibration-free, Particle Size Measurement Using 3D Profile Data”, Edited Sanchidrián \& Singh, Taylor \& Francis Group, London, (2013), pp. 23-32.

[15] W. X. Wang, "Image Analysis of Aggregates", Computers \& Geosciences, vol. 1, no. 25, (1999), pp. 71-81.

[16] W. X. Wang, "Binary Image Segmentation of Aggregates Based on Polygonal Approximation and Classification of Concavities", Pattern Recognition, vol. 10, no. 31, (1998), pp. 1503-1524.

[17] C. W. Liao and Y. S. Tarng, "On-line Automatic Optical Inspection System for Coarse Particle Size Distribution”, Powder Technology, vol. 3, no. 189, (2009), pp. 508-513.

[18] M. N. Ahmed, S. M. Yamany and N. Mohamed, "A Modified Fuzzy C-means Algorithm for Bias Field Estimation and Segmentation of MRI Data”, IEEE Trans. Med. Imaging, vol. 3, no. 21, (2002), pp. 193-199.

[19] J. Egger, B. Freisleben and C. Nimsky, "Template-cut: a Pattern-based Segmentation Paradigm”, Nature Scientific Reports, vol. 2, (2012), pp. 420.

[20] A. W. C. Liew, S. H. Leung and W. H. Lau, "Fuzzy Image Clustering Incorporating Spatial Continuity", Image Signal Process, vol. 2, no. 147, (2000), pp. 185-192. 


\section{Authors}

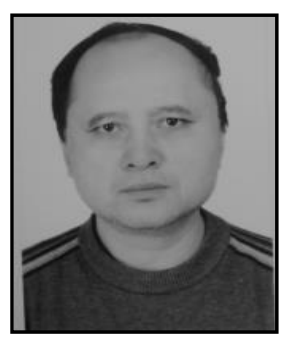

Wang Weixing, Professor in Information engineering. Now he is a visiting professor at Fuzhou University, and his interests involve Image processing and analysis, Pattern recognition and Computer Vision.

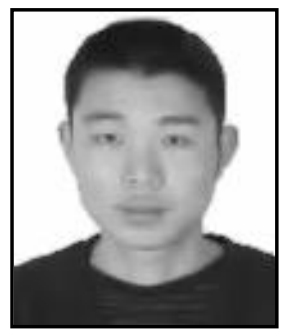

Liang Yanjie, he received B.E. in Electronic Science \& Technology from Fuzhou University (2013). Now he is a student at School of Physics \& Information Engineering, Fuzhou University. His current research interests include image processing, pattern recognition, and computer vision.

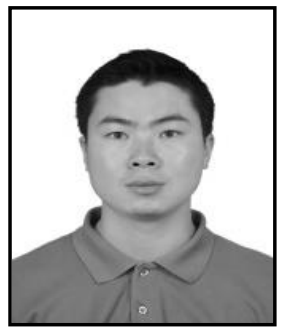

Liao Yipeng, $\mathrm{He}$ is working as teacher at College of Physics and Information Engineering,Fuzhou University,China. Currently, he is pursuing his Ph.D. degree at Fuzhou University. His research interests include Image Analysis and Processing. 